\title{
Thermal Properties of the Tetraquark $X(3872)$ in Diquark-antidiquark Approach
}

\author{
E. Veli Veliev* \\ Department of Physics, Kocaeli University, 41380 Izmit, Turkey \\ Education Faculty, Kocaeli University, 41380 Izmit, Turkey \\ E-mail: elsen@kocaeli.edu.tr

\section{S. Günaydın} \\ Department of Physics, Kocaeli University, 41380 Izmit, Turkey \\ E-mail: sehergnydn@hotmail.com

\section{H. Sundu} \\ Department of Physics, Kocaeli University, 41380 Izmit, Turkey \\ E-mail: hayriye.sundu@kocaeli.edu.tr
}

\begin{abstract}
The thermal features of the $X(3872)$ state with the quantum numbers $J^{P C}=1^{++}$are investigated by considering it as a diquark-antidiquark bound state. The nature of this exotic resonance is explored using QCD sum rule method. The numerical calculations demonstrate that in the low temperature region the mass and current coupling constant of $X(3872)$ is insensitive to temperature, but decreases significantly before the critical temperature. At the deconfinement temperature the current coupling constant and mass attains approximately to $34 \%$ and $74 \%$ of their vacuum values, respectively. The zero-temperature results are in good agreement with the experimental measurements and other theoretical studies presented in the literature.
\end{abstract}

XVII International Conference on Hadron Spectroscopy and Structure

25-29 September, 2017

University of Salamanca, Salamanca, Spain

${ }^{*}$ Speaker. 


\section{Thermal Sum Rule for the $X(3872)$ State}

Exploration of non-perturbative properties of QCD is one of the difficult and intriguing research areas of the strong interactions. The investigation of the hadron spectrum can play an important role in achieving this goal. According to QCD, not only traditional mesons and baryons, but also exotic particles such as glueballs, hybrids and multiquarks states can be observed. During last decade, observation of charmonium-like, bottomonium-like and the pentaquark states in experiments and their detailed examination revealed significant information about exotic particles. The observation of the unconventional states with structures beyond the standard quark model motivated different theoretical interpretations such as molecule and diquark-antidiquark models. The examination of exotic particles is very important to understand the heavy ion collisions. But to this end, we need to know the thermal properties of these particles. For this reason, it is important to examine the exotic particles in the medium.

In 2003 the Belle collaboration announced the discovery of the resonance X(3872) [1]. This particle was confirmed shortly thereafter by the CDF [2], D0 [3], BaBar [4, 5] and LHCb [6] collaborations. In this article we use the Thermal QCD sum rules method to study the $X(3872)$ resonance with quantum numbers $J^{P C}=1^{++}$. We consider it as a diquark-antidiquark bound state.

To calculate the mass and current coupling of the $X(3872)$ state in the framework of the thermal QCD sum rules we start from the correlation function

$$
\Pi_{\mu v}(q, T)=i \int d^{4} x e^{i q \cdot x}\left\langle\mathscr{T}\left\{J_{\mu}(x) J_{v}^{\dagger}(0)\right\}\right\rangle,
$$

where $\mathscr{T}$ indicates the time ordering operator. In the diquark-antidiquark model, the interpolating current of the $X(3872)$ state $J_{\mu}(x)$ is expressed by the following expression

$$
J_{\mu}(x)=\frac{i \varepsilon_{a b c} \varepsilon_{d e c}}{\sqrt{2}}\left\{\left[q_{a}^{T}(x) C \gamma_{5} c_{b}(x)\right]\left[\bar{q}_{d}(x) \gamma_{\mu} C \bar{c}_{e}^{T}(x)\right]+\left[q_{a}^{T}(x) C \gamma_{\mu} c_{b}(x)\right]\left[\bar{q}_{d}(x) \gamma_{5} C \bar{c}_{e}^{T}(x)\right]\right\}
$$

where $q$ is one of the light $u$ or $d$ quarks. In Eq. (1.2) $a, b, c, d, e$ are color indexes and $C$ is the charge conjugation operator.

In order to derive QCD sum rules we first calculate the correlation function in terms of the physical degrees of freedom. Performing integral over $x$ in Eq. (1.1), we get

$$
\Pi_{\mu \nu}^{\text {Phys }}(q, T)=\frac{\left\langle 0\left|J_{\mu}\right| X(q)\right\rangle_{T}\left\langle X(q)\left|J_{v}^{\dagger}\right| 0\right\rangle_{T}}{m_{X}^{2}(T)-q^{2}}+\ldots,
$$

where $m_{X}(T)$ is the temperature-dependent mass of $X(3872)$ and dots stand for the contributions of the higher resonances and continuum states. We define the temperature-dependent current coupling $f_{X}(T)$ through the matrix element

$$
\left\langle 0\left|J_{\mu}\right| X(q)\right\rangle_{T}=f_{X}(T) m_{X}(T) \varepsilon_{\mu},
$$

with $\varepsilon_{\mu}$ being the polarization vector of the $X(3872)$ state. Then in terms of $m_{X}(T)$ and $f_{X}(T)$, the correlation function can be written in the form

$$
\Pi_{\mu \nu}^{\text {Phys }}(q, T)=\frac{m_{X}^{2}(T) f_{X}^{2}(T)}{m_{X}^{2}(T)-q^{2}}\left(-g_{\mu \nu}+\frac{q_{\mu} q_{v}}{m_{X}^{2}(T)}\right)+\ldots
$$


The required sum rules can be obtained by using coefficient, $\Pi_{1}^{\text {Phys }}(q, T)$, corresponding to the structure $-g_{\mu \nu}$. After performing the Borel transformation, the physical side is found as

$$
\mathscr{B}_{q^{2}} \Pi_{1}^{\mathrm{Phys}}(q, T)=m_{X}^{2}(T) f_{X}^{2}(T) e^{-m_{X}^{2}(T) / M^{2}} .
$$

The correlation function in QCD side, $\Pi_{\mu \nu}^{\mathrm{QCD}}(q, T)$, has to be determined employing the quarkgluon degrees of freedom. To this end, we contract the heavy and light quark fields and find for the correlation function $\Pi_{\mu \nu}^{\mathrm{QCD}}(q, T)$ in the diquark-antidiquark picture the following expression:

$$
\begin{aligned}
\Pi_{\mu \nu}^{\mathrm{QCD}}(q, T) & =-\frac{i}{2} \int d^{4} x e^{i q \cdot x} \varepsilon \tilde{\varepsilon} \varepsilon^{\prime} \tilde{\varepsilon}^{\prime}\left\langle\left\{\operatorname{Tr}\left[\gamma_{5} \widetilde{S}_{q}^{a a^{\prime}}(x) \times \gamma_{5} S_{c}^{b b^{\prime}}(x)\right] \operatorname{Tr}\left[\gamma_{\mu} \widetilde{S}_{c}^{e^{\prime} e}(-x) \gamma_{v} S_{q}^{d^{\prime} d}(-x)\right]\right.\right. \\
& +\operatorname{Tr}\left[\gamma_{\mu} \widetilde{S}_{c}^{e^{\prime} e}(-x) \gamma_{5} S_{q}^{d^{\prime} d}(-x)\right] \operatorname{Tr}\left[\gamma_{v} \widetilde{S}_{q}^{a a^{\prime}}(x) \gamma_{5} S_{c}^{b b^{\prime}}(x)\right] \\
& +\operatorname{Tr}\left[\gamma_{5} \widetilde{S}_{q}^{a^{\prime} a}(x) \gamma_{\mu} S_{c}^{b^{\prime} b}(x)\right] \operatorname{Tr}\left[\gamma_{5} \widetilde{S}_{c}^{e^{\prime} e}(-x) \gamma_{v} S_{q}^{d^{\prime} d}(-x)\right] \\
& \left.\left.+\operatorname{Tr}\left[\gamma_{v} \widetilde{S}_{q}^{a a^{\prime}}(x) \times \gamma_{\mu} S_{c}^{b b^{\prime}}(x)\right] \operatorname{Tr}\left[\gamma_{5} \widetilde{S}_{c}^{e^{\prime} e}(-x) \gamma_{5} S_{q}^{d^{\prime} d}(-x)\right]\right\}\right\rangle_{T}
\end{aligned}
$$

In Eq. (1.7) we use the notation

$$
\widetilde{S}_{c(q)}^{i j}(x)=C S_{c(q)}^{i j T}(x) C,
$$

with $S_{q}^{i j}(x)$ and $S_{c}^{i j}(x)$ being the light and heavy quark propagators at the finite temperature, respectively.

The correlation function $\Pi_{\mu \nu}^{\mathrm{QCD}}(q, T)$ can be decomposed over the Lorentz structures

$$
\Pi_{\mu \nu}^{\mathrm{QCD}}(q, T)=\Pi_{0}^{\mathrm{QCD}}\left(q^{2}, T\right) \frac{q_{\mu} q_{v}}{q^{2}}+\Pi_{1}^{\mathrm{QCD}}\left(q^{2}, T\right)\left(-g_{\mu v}+\frac{q_{\mu} q_{v}}{q^{2}}\right),
$$

where $\Pi_{0}^{\mathrm{QCD}}\left(q^{2}, T\right)$ and $\Pi_{1}^{\mathrm{QCD}}\left(q^{2}, T\right)$ are invariant functions which related to the scalar and vector currents, respectively. The QCD sum rules for the mass and current coupling are derived after fixing the same structures in both $\Pi_{\mu \nu}^{\mathrm{Phys}}(q, T)$ and $\Pi_{\mu \nu}^{\mathrm{QCD}}(q, T)$. As in the physical side of the sum rule, in its QCD side the structure containing $-g_{\mu \nu}$ has been taken into account. In the case $\vec{q}=0$, i.e. in the rest frame of the resonance particle, we can write $\Pi_{1}^{\mathrm{QCD}}\left(q_{0}^{2}, T\right)$ as the dispersion integral,

$$
\Pi_{1}^{\mathrm{QCD}}\left(q_{0}^{2}, T\right)=\int_{4 m_{c}^{2}}^{s_{0}(T)} \frac{\rho^{\mathrm{QCD}}(s, T)}{s-q_{0}^{2}} d s+\ldots
$$

where $\rho^{\mathrm{QCD}}(s, T)$ is the corresponding spectral density. The main question of this investigation is calculation of $\rho^{\mathrm{QCD}}(s, T)$. In the present work we include into our sum rules the quark, gluon and mixed condensates up to six dimensions. Analytic results of our calculations are given in [7].

\section{Conclusion and Discussion}

Beside input parameters, we need the temperature dependent quark and gluon condensates, as well as the temperature dependent energy density (in details see [7]). The sum rules for the mass and coupling constant contain two auxiliary parameters, namely continuum threshold $s_{0}$ and Borel mass parameter $M^{2}$. The continuum threshold $s_{0}$ is not completely arbitrary and it is related to the energy of the first exited state of the meson. The interval of continuum threshold is chosen $s_{0}=$ 
$(16.5-17.0) \mathrm{GeV}^{2}$. For the Borel mass parameter, we take the interval $M^{2}=(3.5-5.5) \mathrm{GeV}^{2}$, in which the physical quantities show relatively weak dependence on it.

The final task is to investigate the variations of the mass and current coupling of the $X(3872)$ state with respect to temperature. We obtained that mass and coupling constant of $X(3872)$ obey following fit function :

$$
y(T)=A+B e^{\lambda T} .
$$

The values of the parameters $A, B$ and $\lambda$ are given in Table 1 .

\begin{tabular}{|c|c|c|c|}
\hline \hline $\mathrm{y}(\mathrm{T})$ & $A$ & $B$ & $\lambda\left(\mathrm{GeV}^{-1}\right)$ \\
\hline$m_{X(3872)}$ & $3.90 \mathrm{GeV}$ & $-1.67 \times 10^{-4} \mathrm{GeV}$ & 46.10 \\
\hline$f_{X(3872)}$ & $0.32 \times 10^{-2} \mathrm{GeV}^{4}$ & $-1.42 \times 10^{-5} \mathrm{GeV}^{4}$ & 26.82 \\
\hline \hline
\end{tabular}

Table 1: Parameters appearing in fit function.

At $T=0$, values of the mass and current coupling for $X(3872)$ are obtained as $m_{X(3872)}=$ $(3885 \pm 78) \mathrm{GeV}, f_{X(3872)}=(0.31 \pm 0.11) \times 10^{-2} \mathrm{GeV}^{4}$. Our investigation shows that the mass and current coupling remain unchanged with the variation of temperature up to $T \cong 110 \mathrm{MeV}$, but after this point, they start to diminish with increasing temperature. At the deconfinement temperature the current coupling and mass attain approximately to $34 \%$ and $74 \%$ of their vacuum values, respectively. Considerable decreasing in the values of mass and coupling parameter can be considered as a sign of the quark-gluon plasma (QGP) phase transition. Also, their dependence on temperature obtained in the present work can be used to analyze results of heavy ion collision experiments.

\section{Acknowledgement}

This work has been supported in part by the Kocaeli University through the grant BAP 2017/018.

\section{References}

[1] S. K. Choi et al. [Belle Collaboration], Phys. Rev. Lett. 91, 262001 (2003).

[2] D. Acosta et al. [CDF Collaboration], Phys. Rev. Lett. 93, 072001 (2004).

[3] V. M. Abazov et al. [D0 Collaboration], Phys. Rev. Lett. 93, 162002 (2004).

[4] B. Aubert et al. [BaBar Collaboration], Phys. Rev. D 71, 071103 (2005).

[5] B. Aubert et al. [BaBar Collaboration], Phys. Rev. Lett. 95, 142001 (2005).

[6] R. Aaij et al. [LHCb Collaboration], Nucl. Phys. B 886, 665 (2014).

[7] E. Veli Veliev, S. Günaydın and H. Sundu, arXiv:1707.03714 [hep-ph]. 